\title{
Cognitive Predictors of Medical Decision-Making Capacity in Traumatic Brain Injury
}

\author{
Laura E. Dreer, Michael J. DeVivo, Thomas A. Novack, Sara Krzywanski, and Daniel C. Marson \\ University of Alabama at Birmingham
}

\begin{abstract}
Objective: To identify cognitive predictors of medical decision-making capacity (MDC) in participants with moderate to severe traumatic brain injury (TBI). Participants: At baseline, participants were 34 adults with TBI and 20 healthy adults. At 6-month follow-up, participants were 24 adults with TBI and 20 healthy adults. Main Outcome Measures: Participants were administered the Capacity to Consent to Treatment Instrument (CCTI) and neuropsychological test measures. Multivariate cognitive predictor models were developed for CCTI consent abilities/standards (S) of understanding (S5); reasoning (S4); and appreciation (S3). Results: At baseline, short-term verbal memory and semantic fluency predicted TBI group performance on understanding (S5); short-term verbal memory and attention predicted performance on reasoning (S4); and working memory predicted performance on appreciation (S3). At 6 month follow-up, executive function, verbal processing speed, and working memory predicted TBI performance on understanding (S5); working memory and short-term memory predicted reasoning (S4); and basic executive functioning predicted appreciation (S3). Conclusions: Multiple cognitive functions are associated with acute impairment and partial recovery of MDC in patients with TBI. Short-term verbal memory predicted consent capacity of TBI participants at the time of acute inpatient hospitalization, while executive functioning and working memory predicted improved capacity at six-month follow-up.
\end{abstract}

Keywords: medical decision-making capacity, competency, traumatic brain injury, neurocognitive functioning

Impairment and recovery of competency is a topic of clinical importance and increasing scientific interest in the field of traumatic brain injury (TBI) (Marson et al., 2005; Mukherjee \& McDonough, 2006; Simon, 1994; Wong, 1998; Wong, Clare, Gunn, \& Holland, 1999). Of particular importance to rehabilitation professionals is the question of whether a patient with TBI has the capacity to consent to treatment (medical decision-making capac-

Laura E. Dreer, Department of Ophthalmology, University of Alabama at Birmingham; Michael J. DeVivo and Thomas A. Novack, Department of Physical Medicine and Rehabilitation, University of Alabama at Birmingham; Sara Krzywanski and Daniel C. Marson, Department of Neurology and Alzheimer's Disease Research Center, University of Alabama at Birmingham.

Study supported primarily by a UAB Traumatic Brain Injury Care System grant (NIDDR, H133A980010) (Novack, PI) and related project "Medical and Financial Decision Making Following TBI" (Marson, PI). Other sources of support include the National Institute on Child Health and Human Development (T32 HD07420) (Richards, PI) and (1R01 HD053074) (Marson, PI), an Alzheimer's Disease Research Center grant (NIH, NIA 1P50 AG16582) (Marson, PI), the Alzheimer's Disease Cooperative Study (NIH, NIA U01 AG 10483-12) (Aisen, PI), National Eye Institute (1 K23 EY017327) (Dreer, PI), the EyeSight Foundation of Alabama (ESFA) (Dreer, PI), and 1RO1 AG021927 (Marson, PI).

The capacity outcome measure used in the study is owned by the UAB Research Foundation (UABRF). Dr. Marson receives royalty income through UABRF.

Address correspondence and requests for reprints to Daniel Marson, JD, $\mathrm{PhD}$, Department of Neurology, Room JT1216, University of Alabama at Birmingham, Birmingham, AL 35233-7340. E-mail: dmarson@uab.edu ity, or MDC). This capacity is an important instrumental activity of daily living (IADL) and is strongly associated with functional independence and personal autonomy (Marson, Ingram, Cody, \& Harrell, 1995). MDC is also a key component of the legal doctrine of informed consent that requires that a valid consent to treatment be informed, voluntary, and competent (Kapp, 1992). MDC can be generally defined as the mental and emotional capacity of a patient to consent to, or refuse, a specific medical intervention (Grisso, 1986; Tepper \& Elwork, 1984) and requires an ability to understand the nature of the information provided as well as the risks and benefits of the options (Mukherjee \& McDonough, 2006).

Medical treatment decisions concerning a patient's treatment consent capacity are very common in TBI and may arise during both acute hospitalization and also the rehabilitation period (Hannay \& Fischer, 2004; Marson et al., 2005; Wong, 1998). A range of emergency medical treatment issues commonly confront patients, families, and clinicians at the time of acute injury for those individuals suffering from a severe or moderate TBI, including sustaining life support, artificial respiration, artificial nutrition and hydration, cardiopulmonary resuscitation, orthopedic decisions, antibiotic use, managing or changing medications, and use of advance directives (Bontke, Dolan, \& Ivanhoe, 1994; Childs \& Cranford, 1997; Cope, 1989; Jennett, 1997; Phipps, 1998; Simon, 1994; Zink, 2001). In addition, secondary complications such as seizures, hydrocephalus, infections, vascular injuries, and bedsores may require medical interventions and decisions. These immediate medical decisions are often made by clinicians and family members. However, during the rehabilitation period, when the individual with a TBI becomes more oriented and alert, new med- 
ical questions arise such as whether or not to participate in therapies, take medications, undergo further orthopedic surgeries, or ambulate independently. Questions may also develop concerning the TBI survivor's capacity to make medical decisions for others (i.e., TBI survivor's minor children, spouse, or elderly parent). These are issues that TBI survivors increasingly may want to participate in.

Currently there is little empirical research on competency loss and recovery in TBI that can inform clinical practice (Marson et al., 2001). Our group recently empirically studied MDC longitudinally in a sample of adult patients with moderate and severe TBI (Marson et al., 2005). The study found that at the time of acute hospitalization, complex treatment consent abilities of appreciation, reasoning, and understanding were significantly impaired in patients with TBI, whereas simple consent abilities of evidencing a choice and making the reasonable choice remained relatively intact. At 6-month follow-up, TBI patients showed substantial recovery of the reasoning consent ability, but only partial recovery of the appreciation and understanding consent abilities. The study suggested the importance in the rehabilitation setting of serial evaluations of MDC in patients with TBI.

An ongoing clinical issue and problem for rehabilitation clinicians is determining whether or not a patient with TBI has recovered sufficient decisional capacity to permit resumption of autonomous medical decision-making (Hillier, Sharpe, \& Metzer, 1997; Wong et al., 1999). In this regard, neuropsychological studies of competency can make both significant scientific contributions and also support clinician judgments of future consent capacity. Such studies can illuminate the relationship of specific cognitive changes both to initial loss and also subsequent recovery of consent capacity in TBI, alert clinicians to specific cognitive deficits that threaten competency, and facilitate use of clinical interventions that support the competency of patients with specific cognitive deficits (Marson, Chatterjee, Ingram, \& Harrell, 1996). Neuropsychological mechanisms of MDC impairment and loss have been identified in dementia populations, in particular in patients with Alzheimer's disease and Parkinson's disease (Dymek, Atchison, Harrell, \& Marson, 2001; Earnst, Marson, \& Harrell, 2000; Griffith, Dymek, Atchison, Harrell, \& Marson, 2005; Marson, Cody, Ingram, \& Harrell, 1995; Marson et al., 1996; Moye, Karel, Gurrera, \& Azar, 2006). These studies have significantly advanced our understanding of competency as a construct.

In contrast, very little is known about neurocognitive predictors associated with MDC impairment and recovery in patients with TBI (Marson et al., 2005; Reid-Proctor, Galin, \& Cummings, 2001). This represents an important knowledge gap, as neurocognitive change and outcome may be useful predictors to rehabilitation clinicians seeking to determine whether a patient with TBI can resume medical decision-making. In the present study, we investigate cognitive predictors of MDC in TBI patients at time of acute injury and at 6-month follow-up using an established capacity assessment instrument incorporating a series of consent abilities.

\section{Method}

\section{Participants}

Thirty-four participants with moderate to severe TBI were recruited through the TBI inpatient service at the University of
Alabama at Birmingham (UAB) as part of a larger National Institute on Disability and Rehabilitation Research Traumatic Brain Injury Model Systems (TBIMS) grant. Criteria for moderate to severe TBI included an initial Glasgow Coma Scale (GCS) (Teasdale \& Jennett, 1974) of 12 or below, posttraumatic amnesia (PTA) continuing for a day or more, or objective signs of structural lesion on CT or MRI scan (i.e., contusion or hematoma). Exclusions included patients with a history of any other disease or conditions potentially affecting cognition, such as psychiatric disturbance (with the exception of mild depression), cerebrovascular disease, or other neurological diseases (excluding headache). At 6-month follow-up, the sample consisted of 24 TBI participants reflecting attrition over time. The attrition rate was approximately $30 \%$ from baseline to 6-month follow-up in the TBI group.

Twenty healthy, adult men and women participants (i.e., control group) were recruited through advertisements at UAB and in a Birmingham newspaper. This group of healthy men and women were free of any diseases or conditions that could potentially affect cognition, including psychiatric disturbance (except mild depression), substance abuse, cerebrovascular disease, or other neurological diseases (excluding headache). None of these men or women were taking any medications known to significantly affect cognition. Accordingly, they were viewed to represent a group that was cognitively normal for purposes of the study. Recruitment was also selective and involved individual demographic matching of control participants with existing participants with TBI based on age, gender, ethnicity, and educational level.

Informed consent was obtained from all controls, and from all TBI participants or their participant caregivers/legally authorized representatives (LARs), in accordance with approval from local IRB procedures. All participants received $\$ 50.00$ compensation for each of the two study visits.

\section{Measures}

Capacity assessment measure. The Capacity to Consent to Treatment Instrument (CCTI; Marson, Ingram, et al., 1995) was used to assess MDC. The CCTI consists of two clinical vignettes presenting different hypothetical medical problems (cardiovascular disease, brain tumor) along with treatment options with associated risks and benefits (Marson \& Briggs, 2001). The vignettes are read aloud to the participants, who are asked to simultaneously read the vignettes. The vignettes require participants to consider the medical problem and symptoms, the diagnosis, and the risks and benefits of the different treatment options presented. After the vignette information is presented, the participants answer questions designed to test MDC under the well-established and accepted consent abilities, or standards (S) (Grisso, 1986; Grisso \& Appelbaum, 1995; Marson, Ingram, et al., 1995; Roth, Meisel, \& Lidz, 1977):

- Simply evidencing/communicating a treatment choice (S1);

- Appreciating the personal consequences of a treatment choice (S3);

- Reasoning about treatment/providing rational reasons for a treatment choice (S4); and

- Understanding the treatment situation and choices (S5).

In addition to these four standards, the CCTI evaluates an additional consent ability described as making the "reasonable" treatment choice [S2] (Roth, Meisel, \& Lidz, 1977). This consent 
ability concerns whether a subject can make a treatment decision congruent with the decision that a "reasonable" person in like circumstances would make. Making the "reasonable" treatment choice standard [S2] is not an accepted standard for evaluating MDC because of concerns about arbitrariness in determining what constitutes a "reasonable choice" (Tepper \& Elwork, 1984). Making the "reasonable" treatment choice [S2] is thus referenced in brackets to distinguish it from the other four established standards. However, making the "reasonable" treatment choice [S2] remains useful as an experimental standard for understanding treatment preferences of patients with neurocognitive disorders (Dymek et al., 2001).

The CCTI has a detailed and well-operationalized quantitative scoring system for each vignette standard and its constituent questions. Subject responses to the CCTI questions are tape recorded and then transcribed to ensure a high degree of scoring accuracy. Scoring is carried out using a standardized scoring protocol. For each CCTI standard, performance scores are summed across the two vignettes to create a composite variable (e.g., S5 variable $=\mathrm{S} 5$ Vignette A score + S5 Vignette B score). Procedures for identifying capacity outcomes on the CCTI standards are discussed below in the statistical methods section.

The CCTI has established reliability and validity in assessing consent capacity in older adult controls, patients with mild and moderate Alzheimer's disease, and patients with Parkinson's disease and dementia (Dymek et al., 2001; Marson, Ingram, et al., 1995). In prior work with controls and mild and moderate AD patients, three trained raters achieved high inter-rater reliability for standards with interval scales $(r=.83, p<.0001)($ S3-S5) and also categorical scales ( $>96 \%$ agreement) (S1, [S2]) (Marson, Ingram, et al., 1995; we also treat $\mathrm{S} 1$ as an interval scale).

The CCTI has shown construct and convergent neuropsychologic validity in empirical clinical studies in patients with $\mathrm{AD}$ and PD (Dymek et al., 2001; Griffith et al., 2005; Marson et al., 1996; Marson, Cody, et al., 1995; Marson, Ingram et al., 1995). The CCTI has also demonstrated a coherent two factor structure (verbal conceptualization, verbal memory) in a dementia sample $(n=82)$ (Dymek, Marson, \& Harrell, 1999).

\section{Neuropsychological Test Battery}

Neuropsychological measures commonly used as part of the UAB TBIMS neuropsychological test battery and representing cognitive domains linked conceptually to competency were administered to participants (Dymek et al., 1999; Griffith et al., 2005; Moye, Karel, Gurrera, \& Azar, 2006; Okonkwo et al., 2008; Ponsford, Draper, \& Schonberger, 2008). In addition, supplemental neuropsychological measures sensitive to capacity were administered. Specifically, the battery comprised the after cognitive domains and tests.

Orientation. Orientation was measured using information and orientation items of the Galveston Orientation and Amnesia Test (GOAT; Levin, O’Donnell, \& Grossman, 1979). This measure was developed as a prospective measure of emergence from posttraumatic amnesia. Specific items include orientation to person, place, time, and situation. The total number of error points was used as the score for the current analysis.

Language comprehension. Propositional auditory language comprehension, relying upon organization, manipulation, and ex- ecution of simple and complex verbal commands, was measured using a short form of the Token Test from the Multilingual Aphasia Examination (Benton \& Hamsher, 1989). The Token Test also tests components of executive functioning, such as selective attention, freedom from distractibility, sequencing, and inhibition of responses, as demonstrated in a previous study of medical decision making capacity in Alzheimer's disease (Marson, 2001).

Attention/concentration. Attention and concentration were measured using the Digit Span subtest of the Wechsler Adult Intelligence Scale-Revised (WAIS-R; Wechsler, 1981). Additionally, working memory and short-term attention were evaluated using the Arithmetic subtest of the Wechsler Adult Intelligence Scale-Third Edition (WAIS-III; Wechsler, 1997).

Visuospatial skills. Spatial construction skills were measured with the Block Design subtest of the Wechsler Adult Intelligence Scale-Revised (WAIS-R; Wechsler, 1981). Visual discrimination was assessed using the Benton Visual Discrimination Test (BVDT; Benton, Hamsher, Varney, \& Spreen, 1983).

Information processing speed. Simple visuomotor planning and tracking was assessed using the Trail Making Test A test from the Halstead Reitan battery (Reitan \& Wolfson, 1993) and the Symbol Digit Modalities Test (Oral and Written) (SDMT; Smith, 1982).

Memory. Short-term verbal memory and learning were measured using the Logical Memory I subtest of the Wechsler Memory ScaleRevised (WMS-R; Wechsler, 1987) and the Rey Auditory Verbal Learning Test (RAVLT; Rey, 1964; Taylor, 1959). Delayed verbal recall was measured using the Logical Memory II subtest of the WMS-R as well as the RAVLT. Short and long-term visual memory were evaluated using the 7/24 Spatial Recall Test (Rao, 1990).

Executive function. Simple executive abilities were measured using the Executive Interview (EXIT-25; Royall, Mahurin, \& Gray, 1992). More complex visuospatial tracking and planning was measured using the Trail Making B test from the Halstead Reitan battery (Reitan \& Wolfson, 1993). Concept formation and mental flexibility were assessed using the Wisconsin Card Sorting Test (WCST; Berg, 1948; Grant \& Berg, 1948), and phonemic and semantic word fluency were measured using Controlled Oral Word Association Test form the Multilingual Aphasia Examination (COWA; Benton \& Hamsher, 1989), Animal Naming (Lezak, Loring, Howieson, Fischer, \& Hannay, 2004), Vegetables (Lezak et al., 2004), and Clothing (Lezak et al., 2004).

Fine motor functioning. The Grooved Pegboard (Heaton, Grant, $\&$ Matthews, 1991) was used to assess fine motor functioning.

\section{Procedures}

All study participants underwent baseline (Time 1) and 6-month follow-up (Time 2) study assessments. The baseline assessment for TBI participants was conducted at the end of the acute inpatient hospitalization at Spain Rehabilitation Center, after TBI participants' confusional states and posttraumatic amnesia had cleared. TBI participants received a follow-up assessment 6-months after the date they incurred their TBI (with a scheduling window of 4 weeks before/after this date). The healthy adult participants completed an identical baseline assessment and a 6-month follow-up assessment at the UAB Department of Neurology.

At each study visit, participants were administered the CCTI and a neuropsychological battery. Administration of the two CCTI vignettes 
was counterbalanced to control for possible order effects. Participants' responses on the CCTI vignettes were audiotaped and transcribed to ensure accurate scoring. The CCTI vignettes were scored according to a standardized protocol (Marson, Ingram et al., 1995).

\section{Data Analyses}

Performance on neuropsychological test measures. Group performance on the neurocognitive variables was compared using a mixed factors MANOVA model with a between groups factor (healthy adults, TBI) and a within-groups repeated measures factor of time (Time 1 to Time 2). Subsequent univariate planned comparisons were conducted for each neurocognitive measure, as well as an independent-samples t-test to evaluate baseline group differences on the measure, and paired t-tests within each participant group to evaluate change over time.

Performance on CCTI standards. The data analysis approach for the CCTI consent standards has been described elsewhere (Marson et al., 2005) and was very similar to that described above for the neurocognitive measures. Performance on all variables except making the "reasonable" treatment choice [S2] was evaluated using a mixed factors MANOVA model with a between groups factor (control, TBI) and a within-groups repeated measures factor of time. Subsequent univariate planned comparisons were conducted for each standard, as well as an independentsamples t-test to evaluate baseline group differences, and paired t-tests within each participant group to evaluate change over time. On making the "reasonable" treatment choice [S2], which is categorical (scored as correct or incorrect [1 or 0]), we used chi-square to evaluate group differences at baseline and 6-month follow-up.

Correlational and multiple regression analyses at Time 1 and Time 2. For each CCTI standard, we developed three cognitive models: a model of baseline capacity performance (baseline model), a model of capacity performance at 6-month follow-up using 6-month cognitive predictors (6-month model), and a model of capacity performance at 6-month follow-up using baseline cognitive predictors (baseline - 6-month model). Specifically, baseline cognitive predictors were correlated with CCTI performance at baseline (Time 1, $n=34$ ) (baseline model) and 6-month follow-up (Time 2, $n=24$ ) (baseline - 6-month model). Sixmonth follow-up cognitive predictors were correlated with CCTI performance at 6-month follow-up (6-month model). For each group analysis at each time point, we first used univariate correlations (Pearson $r$ ) as a data reduction step to identify a pool of candidate cognitive predictors for each CCTI standard. This predictor pool was then entered into a stepwise multiple linear regression analysis using backward elimination to identify the key multivariate predictors for standards. In this approach, all variables that passed the univariate screening process were entered into the predictive model followed by sequential elimination of the weakest predictor variables until only those making a statistically significant contribution to the model $(p<.05)$ remained.

\section{Results}

\section{Demographic and Mental Status Variables}

Table 1 presents demographic and mental status findings by group. As discussed above, individual demographic matching of
Table 1

Baseline Demographic and Mental Status Variables of Controls and Participants With Moderate to Severe TBI

\begin{tabular}{lccc}
\hline & $\begin{array}{c}\text { Controls } \\
(n=20) \\
X(S D) / N\end{array}$ & $\begin{array}{c}\text { TBI Participants } \\
(n=24)\end{array}$ & \\
& $X(S D) / N$ & $p$ value \\
\hline Age & $32.2(13.4)$ & $30.0(11.7)$ & .633 \\
Education & $13.2(2.2)$ & $12.2(2.5)$ & .800 \\
Gender & 12 & 16 & \\
$\quad$ Men & 8 & 8 & .757 \\
$\quad$ Women & & 19 & \\
Race & 14 & 5 & .509 \\
$\quad$ White & 6 & & \\
African-American & & & \\
GOAT & N/A & $89.6(7.2)^{* *}$ & \\
$\quad$ Time 1 & N/A & $93.0(6.7)^{* * *}$ & \\
$\quad$ Time 2 & N/A & $7.1(3.2)$ & \\
Glasgow Coma Scale & & & \\
\hline
\end{tabular}

${ }^{*} n=23$; one participant missing information; ${ }^{* *} n=22$; two participants missing information.

Note. Gender and race calculated using Fisher's Exact Test; Age and education calculated using Student's t test.

Reprinted with permission from "Impairment and partial recovery of medical decision making capacity in traumatic brain injury: A six month longitudinal study," by D. Marson, L. Dreer, S. Krzywanski, J. Huthwaite, M. DeVivo, and T. Novack, 2005, Archives of Physical Medicine and Rehabilitation, 86, pp. 889-895. Copyright 2005 by Elsevier.

healthy adult participants with TBI participants occurred during the recruitment phase. There were accordingly no group differences in terms of age, gender, racial background, or years of education (Marson et al., 2005). The young adult age of the TBI group was characteristic of this clinical population (Centers for Disease Control and Prevention, 1997). The racial make-up of the two groups was representative of the greater Birmingham area that is approximately $80 \%$ White and $20 \%$ African-American.

For TBI participants, the average GCS score at time of acute hospitalization was $7.1( \pm 3.2)$ and was indicative of moderate to severe injury. TBI participants obtained an average Galveston Orientation and Amnesia Test (GOAT) (Levin, O'Donnell, \& Grossman, 1979) score of 89.6 at baseline and 93.0 at 6-month follow-up. There was no GOAT score available for one TBI participant at baseline and no GOAT score was available for two participants at the 6-month follow-up.

\section{Group Comparisons on CCTI Standards at Baseline (Time 1) and Six-Month Follow-Up (Time 2)}

Table 2 presents group performance on the CCTI standard variables over time. These results are addressed in greater detail elsewhere (Marson et al., 2005). In summary, at hospitalization, TBI participants performed equivalently with controls on standards S1 and [S2] (evidencing choice; reasonable choice), but significantly below controls on appreciation $(\mathrm{S} 3)(p<.001)$, reasoning (S4) $(p<.02)$, and understanding $(\mathrm{S} 5)(p<.001)$. At 6-month follow-up, TBI participants showed significant improvement within group on these three standards (appreciation, reasoning, and understanding), but continued to fall significantly below controls on reasoning (S3) $(p<.006)$ and S5 $(p<.001)$. A group by time interaction emerged on understanding (S5) 
Table 2

Performance on CCTI Standards Across Time by Group

\begin{tabular}{|c|c|c|c|c|c|c|c|c|c|c|}
\hline & Score range & $\begin{array}{l}\text { Controls time } \\
1 X(S D)\end{array}$ & $\begin{array}{l}\text { Controls time } \\
2 X(S D)\end{array}$ & $p^{I}$ & $\begin{array}{l}\text { TBI participants } \\
\text { time } 1 X(S D)\end{array}$ & $\begin{array}{l}\text { TBI participants } \\
\text { time } 2 X(S D)\end{array}$ & $p^{2}$ & Group 1 & Group 2 & $\begin{array}{l}\text { Group/time } \\
\text { interaction }\end{array}$ \\
\hline S1-evidencing choice & $0-4$ & $4.0(0.0)$ & $3.9(0.4)$ & .330 & $3.9(0.4)$ & $4.0(0.0)$ & .328 & .367 & .278 & .163 \\
\hline S3-appreciation & $0-8$ & $6.4(1.1)$ & $6.8(1.4)$ & .248 & $4.5(2.2)$ & $5.4(1.7)$ & .042 & .001 & .006 & .360 \\
\hline S4-reasoning & $0-12$ & $7.7(3.0)$ & $7.8(2.9)$ & .949 & $5.2(3.5)$ & $6.6(3.3)$ & .046 & .014 & .223 & .187 \\
\hline S5-understanding & $0-70$ & $57.2(9.1)$ & $57.6(9.7)$ & .859 & $34.8(11.1)$ & $44.0(12.9)$ & .002 & .001 & .001 & .017 \\
\hline [S2]-reasonable choice & $0-1$ & $1.0(0.2)$ & $1.0(0.2)$ & 1.0 & $0.9(0.3)$ & $1.0(0.2)$ & .328 & .671 & .898 & .367 \\
\hline
\end{tabular}

Note. Time 1 is the baseline assessment; Time 2 is the 6-month follow-up assessment; Group 1 is the control and TBI group difference at Time 1; Group 2 is the control and TBI group differences at time 2 ;

* Control $n=20$; TBI patient $n=24$.

$p^{l}=$ Within group difference across time for controls. $p^{2}=$ Within group difference across time for TBI participants.

Reprinted with permission from "Impairment and partial recovery of medical decision making capacity in traumatic brain injury: A six month longitudinal study," by D. Marson, L. Dreer, S. Krzywanski, J. Huthwaite, M. DeVivo, and T. Novack, 2005, Archives of Physical Medicine and Rehabilitation, 86, pp. 889-895. Copyright 2005 by Elsevier.

$(p<.02)$. The TBI group showed increasing proportions of capable outcomes on all standards over the 6-month period. Control group performance on the CCTI standards showed stability across the two assessments.

\section{Group Comparisons on Neurocognitive Performance at Time 1 and Time 2}

Table 3 details the neuropsychological test results by group. At baseline, the healthy adult group performed significantly better than TBI participants $(p<.05)$ on all measures of cognitive function, consistent with expected cognitive impairment levels for a moderate to severely injured TBI sample at the end of the inpatient course.

At 6-month follow-up, the healthy adult group demonstrated stable or slightly improved scores in comparison to their baseline performance (see column $p^{l}$, Table 3 ). In contrast, the TBI group demonstrated significant within group improvements on almost all measures (see column $p^{2}$ ), reflecting the spontaneous neurocognitive improvement occurring in TBI participants over the 6 months postinjury. The TBI group's improvement across the cognitive measures was also represented by significant group by time interaction effects $(p<.05)$, particularly in the cognitive domains of information processing speed and executive functioning.

However, at 6-month follow-up the TBI group continued to perform significantly below controls on almost all cognitive variables, with the exceptions of visual recall, visual discrimination, and categories achieved on the WCST. Thus, similar to TBI patients' consent capacity performance, only partial neurocognitive improvement occurred over the 6-month postinjury period.

\section{Univariate and Multivariate Models of CCTI Standards in the TBI Group}

Tables 4, 5, and 6 present univariate correlations and multivariate predictor models in the TBI group for the CCTI standards. Table 4 presents the baseline models, Table 5 presents the 6 -month follow-up models, and Table 6 presents the baseline predictor6-month follow-up models. Results are discussed below by each of the CCTI standards.

\section{Evidencing Choice (S1) and Reasonable Choice [S2]}

As discussed above, the TBI group performed equivalently with the healthy adult participants on these standards at both baseline and follow-up, and there was little score variance across groups. Accordingly, cognitive models were unavailable for these elementary and less clinically relevant standards.

\section{Understanding Treatment (S5)}

Understanding treatment is probably the most complex and clinically relevant consent ability (Marson et al., 1996). At baseline, poorer performance on WMS-R Logical Memory I and II $(r=.67, .44$, respectively), Semantic Fluency Composite (animals, fruits/vegetables, clothing; $r=.59)$, RAVLT $(r=.48)$, Token Test $(r=.59)$, EXIT-25 $(r=-.59)$, WAIS-III Arithmetic $(r=.52)$, WAIS-R Digit Span $(r=.45)$, Trails B $(r=-.42)$, and WCST (categories, $r=.40$ ) were significantly associated at the univariate level with poorer performance on understanding treatment (S5) (see Table 4). At the multivariate level, WMS-R Logical Memory I and Semantic Fluency Composite predicted TBI patient performance on understanding treatment (S5). The model accounted for a substantial amount of variance in baseline understanding treatment $(\mathrm{S} 5)$ scores $\left(R^{2}\right.$ cum $\left.=.56, p<.001\right)$.

At 6-month follow-up, measures of executive functioning were primarily associated at the univariate level with performance on understanding treatment (S5) (see Table 5). For example, poorer performance on the Token Test $(r=.67)$, SDMT-oral $(r=.57)$, EXIT-25 $(r=-.49)$, WCST categories completed $(r=.65)$, and Trails B $(r=-.64)$ were related to poorer CCTI performance at 6-month follow-up. The final multivariate model for understanding treatment (S5) at 6-month follow-up consisted of measures of the Token Test, SDMT-Oral, and WAIS-III Arithmetic. The model accounted for a very substantial amount of variance in understanding treatment (S5) scores at 6-month follow-up $\left(R^{2}\right.$ cum $\left.=.72, p<.001\right)$.

We developed a third model for understanding treatment (S5) using baseline cognitive measures to predict S5 performance at 6-month follow-up (see Table 6). Specifically, poorer performance on the Token Tests $(r=.63)$, WAIS-R Digit Span $(r=.72)$, Trails $\mathrm{B}(r=-.61)$, WAIS-III Arithmetic $(r=.60)$, and WCST (cate- 
Table 3

Healthy Adult and TBI Group Comparisons on Neuropsychological Measures at Baseline (Time 1) and Six-Month Follow-Up (Time 2)

\begin{tabular}{|c|c|c|c|c|c|c|c|c|c|}
\hline Measures & $\begin{array}{l}\text { Healthy adults } \\
\text { time } 1 X(S D) \\
\quad N=20\end{array}$ & $\begin{array}{l}\text { Healthy adults } \\
\text { time } 2 X(S D) \\
\qquad N=20\end{array}$ & $p^{l}$ & $\begin{array}{l}\text { TBI time } 1 X \\
\text { (SD) } N=24\end{array}$ & $\begin{array}{l}\text { TBI time } 2 X \\
(S D) N=24\end{array}$ & $p^{2}$ & Group $1 p$ & Group $2 p$ & $\begin{array}{l}\text { Group/time } \\
\text { interaction } p\end{array}$ \\
\hline \multicolumn{10}{|l|}{ Orientation } \\
\hline GOAT & N/A & N/A & - & $88.91(7.35)$ & $93.54(6.66)$ & .041 & - & - & - \\
\hline \multicolumn{10}{|l|}{ Attention } \\
\hline WAIS-R Digit Span & $16.20(4.50)$ & $17.15(4.55)$ & .342 & $12.13(2.94)$ & $14.00(3.36)$ & .001 & .001 & .012 & .330 \\
\hline WAIS-III Arithmetic & $15.40(3.38)$ & $15.20(3.25)$ & 691 & $9.92(2.81)$ & $11.67(3.24)$ & .001 & .001 & .001 & .010 \\
\hline \multicolumn{10}{|l|}{ Information processing speed } \\
\hline Trail making test $\mathrm{A}$ & $29.40(11.10)$ & $27.25(8.79)$ & .317 & $80.79(62.71)$ & $37.71(17.06)$ & .001 & .001 & .017 & .001 \\
\hline SDMT (oral) & $58.10(12.23)$ & $61.45(12.44)$ & .120 & $28.09(13.31)$ & $49.05(13.43)$ & .001 & .001 & .004 & .001 \\
\hline SDMT (written) & $52.95(9.06)$ & $55.85(10.36)$ & .095 & $25.91(12.52)$ & $44.23(15.89)$ & .001 & .001 & .008 & .001 \\
\hline \multicolumn{10}{|l|}{ Short-term memory } \\
\hline WMS-R logical memory I & $27.05(6.13)$ & $30.20(7.27)$ & .054 & $18.13(6.52)$ & $23.13(7.61)$ & .002 & .001 & .003 & .993 \\
\hline RAVLT & $50.70(9.39)$ & $51.21(12.19)$ & .914 & $34.09(12.62)$ & $43.04(11.76)$ & .001 & .001 & .033 & .022 \\
\hline 7/24 spatial recall, total avg. & $5.92(1.22)$ & $6.28(.95)$ & .226 & $5.02(1.30)$ & $5.34(1.27)$ & .266 & .025 & .010 & .834 \\
\hline $7 / 24$ spatial recall, interference & $4.50(1.43)$ & $5.05(1.82)$ & 186 & $3.22(1.54)$ & $3.45(2.26)$ & .554 & .007 & .017 & .786 \\
\hline 7/24 spatial recall, short-delay & $5.95(1.90)$ & $6.65(1.57)$ & 130 & $4.22(1.65)$ & $6.09(1.19)$ & .001 & .003 & .198 & .211 \\
\hline \multicolumn{10}{|l|}{ Delayed memory } \\
\hline WMS-R logical memory II & $24.20(5.93)$ & $27.25(6.53)$ & 012 & $8.65(7.38)$ & $18.52(8.50)$ & .001 & .001 & .001 & .004 \\
\hline 7/24 spatial recall, long-delay & $6.05(1.79)$ & $6.50(1.15)$ & 317 & $4.35(1.77)$ & $5.77(1.72)$ & .033 & .003 & .118 & .200 \\
\hline \multicolumn{10}{|l|}{ Executive functioning } \\
\hline WCST (categories completed) & $5.15(1.35)$ & $5.00(1.65)$ & 634 & $2.17(2.33)$ & $3.79(2.52)$ & .007 & .013 & .073 & .011 \\
\hline Trail making test $\mathrm{B}$ & $74.10(29.52)$ & $63.35(20.60)$ & .106 & $216.67(94.58)$ & $109.58(72.82)$ & .001 & .001 & .009 & .001 \\
\hline EXIT-25 & $5.55(3.00)$ & $4.80(2.38)$ & .284 & $10.25(3.96)$ & $7.67(4.04)$ & .001 & .001 & .008 & .026 \\
\hline COWA-CFL & $14.03(3.21)$ & $14.23(3.50)$ & .728 & $5.60(2.09)$ & $8.81(2.94)$ & .001 & .001 & .001 & .001 \\
\hline Semantic fluency composite & $19.23(4.60)$ & $18.92(4.22)$ & .648 & $12.03(3.60)$ & $14.79(3.50)$ & .001 & .001 & .001 & .002 \\
\hline Token test & $43.10(1.55)$ & $43.90(.91)$ & .046 & $40.77(3.69)$ & $42.17(2.52)$ & .037 & .012 & .006 & .385 \\
\hline \multicolumn{10}{|l|}{ Visuospatial skills } \\
\hline WAIS-R block design & $29.95(13.92)$ & $30.30(12.38)$ & .871 & $15.22(10.39)$ & $23.04(10.77)$ & .001 & .001 & .044 & .012 \\
\hline BVDT & $29.50(2.37)$ & $28.70(3.11)$ & .245 & $25.42(4.94)$ & $28.13(7.61)$ & .025 & .002 & .602 & .049 \\
\hline \multicolumn{10}{|l|}{ Motor functioning } \\
\hline Grooved pegboard & $66.30(17.68)$ & $59.80(8.71)$ & .047 & $148.88(79.64)$ & $89.88(30.33)$ & .001 & .001 & .001 & .002 \\
\hline
\end{tabular}

Note. $\quad p^{1}=$ Within group difference across time for Controls; $p^{2}=$ Within group difference across time for TBI participants.

Group 1 = Healthy adult and TBI group difference at Time 1; Group $2=$ Healthy Adult and TBI group difference at Time $2 ; N=24$.

GOAT $=$ Galveston Orientation and Amnesia Test; WAIS-R Digit Span $=$ Wechsler Adult Intelligence Scale-Revised, Digit Span Subtest; WAIS-III $=$ Wechsler Adult Intelligence Scale-Third Edition, Arithmetic Subtest; Trail Making Test A = Trail Making Test A from the Halstead Reitan Battery; SDMT $($ Oral $)=$ Symbol Digit Modalities Test $($ Oral); SDMT $($ Written $)=$ Symbol Digit Modalities (Written); WMS-R Logical Memory I = Wechsler Memory Scale-Revised, Logical Memory I; RAVLT = Rey Auditory Verbal Learning Test; 7/24 Spatial Recall Test, Total; 7/24 Spatial Recall Test, Total Avg. = 7/24 Spatial Recall Test, Total Score Average; 7/24 Spatial Recall Test, Interference; 7/24 Spatial Recall Test, Short-Delay; WMS-R Logical Memory II = Wechsler Memory Scale-Revised, Logical Memory II; 7/24 Spatial Recall Test, Long-Delay; BVDT = Benton Visual Discrimination Test; WCST (categories completed) $=$ Wisconsin Card Sorting Test; Trail Making Test B = Trail Making Test B from the Halstead Reitan Battery; EXIT-25 = Executive Interview; Semantic Fluency Composite $=$ Sum of Animals, Fruits/Vegetables, and Clothing/3; Token Test $=$ Multilingual Aphasia Examination Token Test; WAIS-III Block Design = Wechsler Adult Intelligence Scale-Third Edition, Block Design Subtest.

gories $r=.56$ ) were among the strongest measures associated at the univariate level with poorer performance on understanding treatment (S5) at 6-month follow-up. The multivariate model consisted of the Token Test, WAIS-R Digit Span, and Trails B. This predictor model also accounted for a very substantial amount of variance in understanding treatment (S5) scores at 6-month follow-up $\left(R^{2}\right.$ cum $\left.=.71, p<.001\right)$.

\section{Reasoning (S4)}

Reasoning about treatment is also a complex consent ability that requires both memory/factual retention and executive and reasoning skills (Marson et al., 1996). At baseline, poorer performance on the WMS-R Logical Memory I and II ( $r=.50$, .45, respectively), WAIS-R Digit Span $(r=.39)$, Token Test $(r=.42)$, and WAIS-III Arithmetic $(r=.39)$ was associated at the univariate level with poorer reasoning (S4) performance (see Table 4). At the multivariate level, WMS-R Logical Memory II and WAIS-R Digit Span predicted TBI performance on reasoning (S4) at baseline. The model accounted for an acceptable amount of variance in baseline reasoning (S4) scores $\left(R^{2}\right.$ cum $=$ $.32, p=.003)$.

At 6-month follow-up, WAIS-III Arithmetic $(r=.54)$, RAVLT $(r=.41)$, WMS-R Logical Memory I $(r=.51)$, and Token Test $(r=.55)$ were primarily associated with poorer performance on reasoning (S4) (see Table 5). The final multivariate model at 6-month follow-up consisted of WAIS-III Arithmetic and to a lesser degree, RAVLT. This model accounted for a substantial amount of variance $\left(R^{2}\right.$ cum $\left.=.48, p=.002\right)$ in reasoning (S4) scores at 6-month follow-up.

Regarding the reasoning (S4) baseline-6-month predictor 
Table 4

Univariate and Multivariate Baseline (Time 1) Cognitive Predictors of TBI Participant Performance $(N=34)$ on CCTI Standards at Baseline (Time 1)

\begin{tabular}{|c|c|c|c|c|c|c|c|}
\hline \multirow[b]{2}{*}{ Standard } & \multirow[b]{2}{*}{ Predictors } & \multicolumn{2}{|c|}{ Univariate } & \multirow[b]{2}{*}{$\beta$} & \multicolumn{2}{|c|}{ Multivariate } & \multirow[b]{2}{*}{$p$} \\
\hline & & $r$ & $p$ & & S.E. $\beta$ & $R^{2}$ cum & \\
\hline S1 Evidencing choice & $<$ No model $>$ & & & - & - & & - \\
\hline [S2] Reasonable choice & $<$ No model $>$ & & & - & - & & - \\
\hline $\begin{array}{l}\text { S3 Appreciating } \\
\text { consequences }\end{array}$ & $\begin{array}{l}\text { WAIS-III arithmetic } \\
\text { (Constant) }\end{array}$ & .49 & .003 & $\begin{array}{l}.382 \\
.812\end{array}$ & $\begin{array}{r}.120 \\
1.212\end{array}$ & & .003 \\
\hline & $\begin{array}{l}\text { Token test } \\
\text { WMS-R logical memory I }\end{array}$ & $\begin{array}{l}.45 \\
.35\end{array}$ & $\begin{array}{l}.01 \\
.05\end{array}$ & & & .24 & .003 \\
\hline S4 Reasoning about treatment & $\begin{array}{l}\text { WMS-R logical memory II } \\
\text { WAIS-R digit span } \\
\text { (Constant) }\end{array}$ & $\begin{array}{l}.45 \\
.39\end{array}$ & $\begin{array}{l}.008 \\
.03\end{array}$ & $\begin{array}{r}.189 \\
.348 \\
-.753\end{array}$ & $\begin{array}{r}.069 \\
.156 \\
1.872\end{array}$ & & $\begin{array}{l}.010 \\
.033\end{array}$ \\
\hline & $\begin{array}{l}\text { WMS-R logical memory I } \\
\text { Token test } \\
\text { WAIS-III arithmetic }\end{array}$ & $\begin{array}{l}.50 \\
.42 \\
.39\end{array}$ & $\begin{array}{l}.03 \\
.02 \\
.02\end{array}$ & & & .32 & -.003 \\
\hline S5 Understanding treatment & $\begin{array}{l}\text { WMS-R logical memory I } \\
\text { Semantic fluency composite } \\
\text { (Constant) } \\
\text { Token test } \\
\text { EXIT-25 } \\
\text { WAIS-III arithmetic } \\
\text { RAVLT } \\
\text { WAIS-R digit span } \\
\text { WMS-R logical memory II } \\
\text { Trails B } \\
\text { WCST (categories) }\end{array}$ & $\begin{array}{r}.59 \\
-.59 \\
.52 \\
.48 \\
.45 \\
.44 \\
-.42 \\
.40\end{array}$ & $\begin{array}{l}.001 \\
.001 \\
.002 \\
.005 \\
.009 \\
.01 \\
.02 \\
.02\end{array}$ & $\begin{array}{r}.938 \\
1.344 \\
1.405\end{array}$ & $\begin{array}{r}.246 \\
.500 \\
5.719\end{array}$ & .56 & $\begin{array}{l}.001 \\
.012 \\
.001\end{array}$ \\
\hline
\end{tabular}

model, poorer performance primarily on baseline Token Test ( $r=$ $.61)$, WAIS-III Arithmetic ( $r=.65)$, and WMS-R Logical Memory I $(r=.61)$ was associated at the univariate level with poorer reasoning (S4) performance at 6-month follow-up (see Table 6). The multivariate model consisted of Token Test and WAIS-III Arithmetic. This model of baseline cognitive predictors accounted for a very substantial amount of reasoning (S4) score variance at 6-month follow-up $\left(R^{2}\right.$ cum $\left.=.61, p<.001\right)$.

\section{Appreciation (S3)}

Appreciating the personal consequences of a treatment decision is a moderately complex consent ability that requires judgment as well as more basic memory/factual retention and executive skills (Marson et al., 1996). At baseline assessment, poorer performance on the WAIS-III Arithmetic subtest $(r=.49)$, Token Test $(r=.45)$, and WMS-R Logical Memory I $(r=.35)$ was related at the univarite level to poorer TBI patient performance on appreciation (S3) (see Table 4). At the multivariate level, WAIS-III Arithmetic emerged as the sole predictor of TBI performance on appreciation (S3). The baseline model accounted for a modest amount of appreciation (S3) score variance $\left(R^{2}\right.$ cum $\left.=.24, p=.003\right)$.

At 6-month follow-up, only Token Test $(r=.49)$ was correlated at the univariate level with performance on appreciation (S3) at 6-month follow-up. This measure was also the sole multivariate predictor and accounted for a modest amount of appreciation (S3) score variance at 6-month follow-up $\left(R^{2}\right.$ cum $\left.=.24, p=.02\right)$ (see Table 5).

Regarding the S3 baseline-6-month predictor model, poorer performance on baseline WAIS-III Arithmetic $(r=.54)$ and WMS-R Logical Memory I $(r=.43)$ was associated at the univariate level with poorer S3 performance at 6-month follow-up (see Table 6). The multivariate model consisted solely of WAIS-III Arithmetic, which accounted for an acceptable amount of S3 score variance $\left(R^{2}\right.$ cum $\left.=.29, p=.007\right)$.

\section{Discussion}

This study presents an initial empirical investigation of neurocognitive predictors of MDC in patients with TBI. Using an MDC assessment measure and a neuropsychological test battery sensitive to TBI and capacity loss, we developed cognitive models for TBI patient performance on the consent abilities of understanding (S5), reasoning (S4), and appreciation (S3). These three standards are the most complex and clinically relevant of the five-treatment consent abilities described in the literature (Marson \& Ingram, 1996; Marson et al., 1996).

We found that multiple cognitive functions are associated with acute impairment and partial recovery of MDC in acutely injured patients with moderate to severe TBI. Short-term verbal memory 
Table 5

Univariate and Multivariate Six-Month Follow-Up (Time 2) Cognitive Predictors of TBI Participant Performance (N = 24) on CCTI Standards at Six-Month Follow-Up (Time 2)

\begin{tabular}{|c|c|c|c|c|c|c|c|}
\hline \multirow[b]{2}{*}{ Standard } & \multirow[b]{2}{*}{ Predictors } & \multicolumn{2}{|c|}{ Univariate } & \multicolumn{3}{|c|}{ Multivariate } & \multirow[b]{2}{*}{$p$} \\
\hline & & $r$ & $p$ & $\beta$ & S.E. $\beta$ & $R^{2}$ cum & \\
\hline S1 Evidencing choice & $<$ No model $>$ & & & - & - & & - \\
\hline [S2] Reasonable choice & $<$ No model $>$ & & & - & - & & - \\
\hline S3 Appreciating consequences & $\begin{array}{l}\text { Token test } \\
\text { (Constant) }\end{array}$ & .49 & .02 & $\begin{array}{r}.333 \\
-8.667\end{array}$ & $\begin{array}{r}.131 \\
5.523\end{array}$ & & $\begin{array}{r}.019 \\
-\end{array}$ \\
\hline S4 Reasoning about treatment & $\begin{array}{l}\text { WAIS-III arithmetic } \\
\text { RAVLT } \\
\text { (Constant) }\end{array}$ & $\begin{array}{l}.54 \\
.41\end{array}$ & $\begin{array}{l}.007 \\
.05\end{array}$ & $\begin{array}{r}.566 \\
.117 \\
-4.979\end{array}$ & $\begin{array}{r}.165 \\
.046 \\
2.808\end{array}$ & .24 & $\begin{array}{l}.02 \\
.003 \\
.019\end{array}$ \\
\hline & $\begin{array}{l}\text { WMS-R logical memory I } \\
\text { Token test } \\
\text { Trails B } \\
\text { SDMT (oral) } \\
\text { Semantic fluency composite } \\
\text { EXIT-25 } \\
\text { WMS-R logical memory II }\end{array}$ & $\begin{array}{r}.51 \\
.55 \\
-.48 \\
.47 \\
.44 \\
-.43 \\
.43\end{array}$ & $\begin{array}{l}.01 \\
.007 \\
.02 \\
.03 \\
.04 \\
.04 \\
.04\end{array}$ & & & .48 & .002 \\
\hline S5 Understanding treatment & $\begin{array}{l}\text { Token test } \\
\text { SDMT (oral) } \\
\text { WAIS-III arithmetic } \\
\text { (Constant) } \\
\text { WCST (categories) } \\
\text { RAVLT } \\
\text { Trails B } \\
\text { WAIS-R block design } \\
\text { 7/24 spatial recall Test (interference) } \\
\text { WAIS-R digit span } \\
\text { Semantic fluency composite } \\
\text { Exit-25 } \\
\text { SDMT (written) } \\
\text { Letter fluency composite } \\
\text { WCST (perseverative responses) } \\
\text { GOAT } \\
\text { WMS-R logical memory II } \\
\text { WMS-R logical memory I }\end{array}$ & $\begin{array}{r} \\
.65 \\
.64 \\
-.64 \\
.64 \\
.53 \\
.51 \\
.49 \\
-.49 \\
.48 \\
.48 \\
-.48 \\
.46 \\
.44 \\
.41\end{array}$ & $\begin{array}{l}.001 \\
.001 \\
.001 \\
.001 \\
.01 \\
.01 \\
.02 \\
.02 \\
.02 \\
.02 \\
.02 \\
.02 \\
.04 \\
.05\end{array}$ & $\begin{array}{r}2.487 \\
.289 \\
1.185 \\
-88.073\end{array}$ & $\begin{array}{r}.698 \\
.129 \\
.531 \\
26.81\end{array}$ & .72 & $\begin{array}{l}.002 \\
.039 \\
.039 \\
.004 \\
.001\end{array}$ \\
\hline
\end{tabular}

was strongly associated with impairments in consent capacity in TBI patients at the time of acute inpatient hospitalization. In contrast, at 6-month follow-up, measures of basic language comprehension, executive function, and working memory were more strongly associated with capacity performance. Baseline measures of basic executive function and working memory also were the best predictors of capacity performance at 6-month follow-up. Overall, the results offer insight into the relationship between different standards of competency and cognitive changes after acute TBI.

Understanding (S5) is perhaps the most clinically relevant consent ability (Marson et al., 1996) and most likely to show impairment in TBI (Marson et al., 2005) and dementia (Marson et al., 1996). The baseline model of understanding (S5) performance consisted of a primary predictor of short-term verbal memory (WMS-R Logical Memory I), and a secondary predictor of semantic fluency (Table 4). Logical Memory I measures immediate auditory verbal recall for narrative verbal material (stories) presented orally to the participant. Semantic word fluency measures speeded access to category specific semantic knowledge (animals, fruits/vegetables, clothing) and also has an executive function component. This baseline model is consistent with our knowledge of TBI and with MDC as a construct. Impairments in short-term memory and executive functions are well-established features of acutely injured patients with moderate to severe TBI (Kersel, Marsh, Havill, \& Sleigh, 2001; Leon-Carrion et al., 1998; Mattson \& Levin, 1990; McDonald, Flashman, \& Saykin, 2002; McDowell, Whyte, \& D'Esposito, 1997; Proctor, Sanchez, \& Wesley, 2000; Vakil, 2005). The understanding consent ability is highly memory intensive and requires the encoding and consolidation of new medical information such as the medical condition and symptoms, and treatment options and their respective risks and benefits. The understanding consent ability also taps conceptualization abilities that are linked to semantic knowledge networks (Marson et al. 1996). The MDC construct as a whole has strong associations to both verbal memory and verbal conceptualization/executive abilities, as revealed in a prior factor analytic study using the CCTI (Dymek et al., 1999).

The 6-month model for understanding (S5) reflected a shift away from verbal memory as the primary predictor (Table 5). The 
Table 6

Univariate and Multivariate Baseline (Time 1) Cognitive Predictors of TBI Participant Performance $(N=24)$ on CCTI Standards at Six-Month Follow-Up (Time 2)

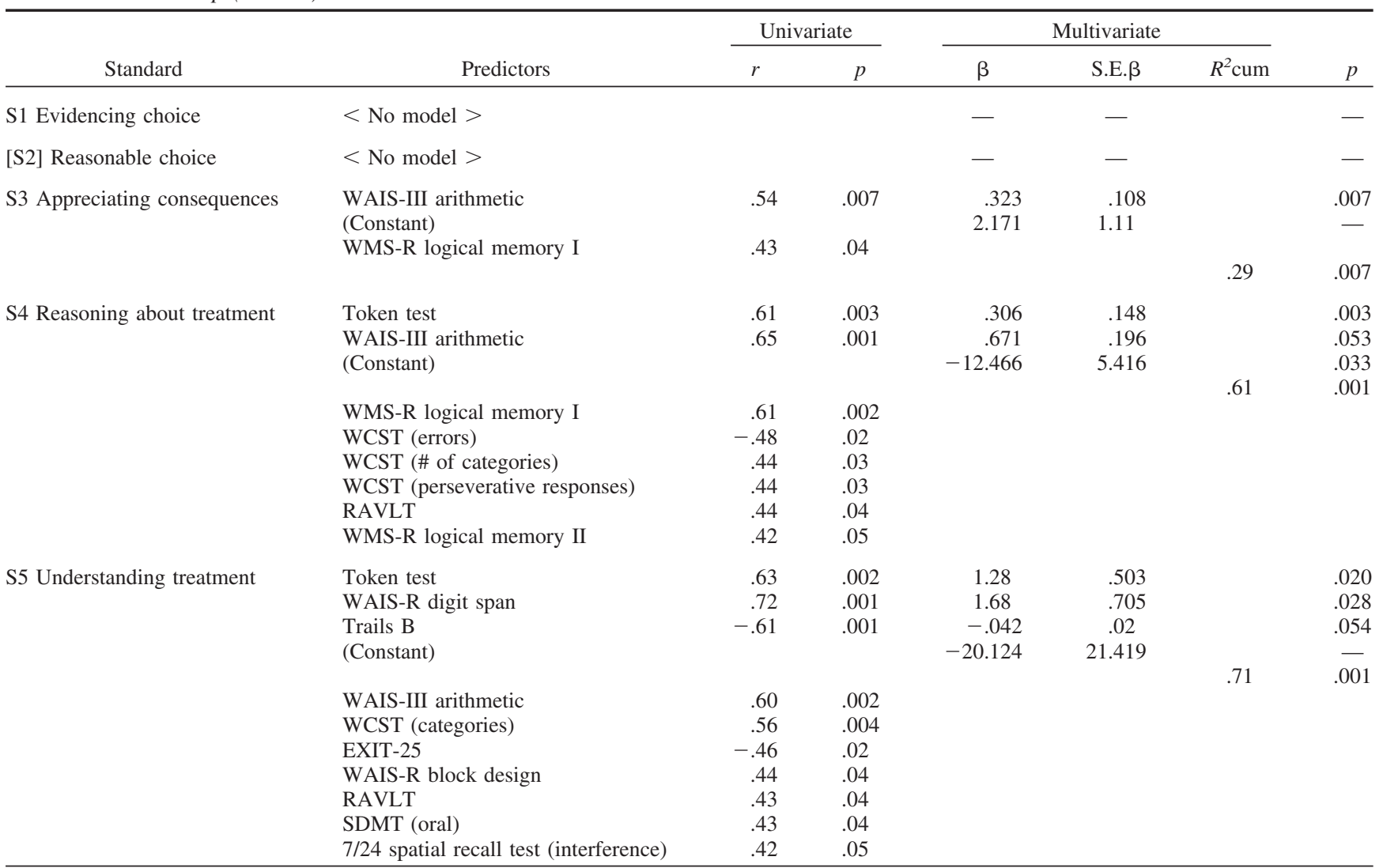

Token Test became a particularly strong predictor at 6-months after injury. Although included as a measure of language comprehension, the Token Test also requires executive skills. Very few of the participants, even at baseline, performed in the impaired range on the Token Test, indicating that difficulty with language comprehension was not a significant factor in this study. The variation in scores noted is interpreted to reflect the executive aspects of the test. At follow-up, the primary cognitive predictor of understanding (S5) performance was Token Test, viewed as a test of executive skills in this study, as well as measures of auditory verbal working memory (WAIS-III Arithmetic) and information processing speed (SDMT-Oral) as secondary predictors. Thus, in the 6-month period after acute TBI, it appears that basic executive functions and working memory replace short-term verbal memory abilities as key predictors of TBI patients' capacity to understand the treatment situation and choices. This finding may reflect the broader clinical reality that a patient's outcome in the year after TBI will be primarily mediated by the patient's basic executive abilities and overall frontal lobe function, and not by memory per se (Leon-Carrion et al., 1998).

The baseline-6-month model of understanding (S5) (Table 6) also reflected a shift from verbal memory predictors to executive function and attentional predictors. The key baseline cognitive predictor of understanding (S5) performance at 6-month follow-up was a measure of basic executive function and language compre- hension (again the Token Test), with secondary predictors of executive function/set flexibility (Trails B), and of auditory verbal attention (WAIS-R Digit Span). The Token Test taps multiple cognitive functions including freedom from distractibility, working memory, and propositional comprehension. In prior capacity studies, it has been shown to function as a measure of simple executive function in cognitively impaired populations such as Alzheimer's disease (Marson, 2001). In the present study, it appears that Token Test performance at the acute hospitalization stage can offer guidance to clinicians concerning the future decisional capacity of an acutely injured patient.

It should be noted that verbal memory measures did not enter into either the 6-month or baseline-6-month multivariate models of understanding (S5), suggesting that the relative importance of verbal memory diminishes as recovery of overall cognitive function, and of decisional capacity, proceeds in TBI.

Reasoning (S4) is a complex and clinically relevant consent ability that requires both memory/factual retention and executive and reasoning skills (Marson et al., 1996). The baseline model of reasoning (S4) performance consisted of a primary measure of short-term verbal memory (Logical Memory I), and a secondary measure of auditory verbal attention (WAIS-R Digit Span). The 6-month cognitive model for reasoning (S4) comprised a primary measure of auditory working memory (WAIS-III Arithmetic) and a secondary measure of high load verbal learning and memory 
(RAVLT). The baseline-6-month cognitive model consisted of a primary measure of basic executive function (Token Test), and a secondary predictor of auditory working memory (WAIS-III Arithmetic). Empirical evidence of deficits in working memory has been repeatedly demonstrated in TBI (McAllister, Flashman, \& Saykin, 2004; Vakil, 2005).

These models for reasoning (S4) indicate that short-term verbal memory is the key cognitive predictor of the reasoning consent ability at the time of acute hospitalization, and to a lesser extent during the initial 6-month recovery period. Similar to understanding (S5), at 6-month follow-up basic executive and working memory abilities begin to emerge as predominant univariate and multivariate predictors of reasoning (S4). This finding again arguably reflects the increasing contribution of frontal and executive processes to everyday functioning in TBI survivors in the initial years postinjury (Leon-Carrion et al., 1998).

Appreciation (S3) is a moderately complex and also clinically relevant consent ability that requires personal judgment as well as memory and executive skills (Marson et al., 1996). The CCTI test items for appreciation (S3) seek to tap participants' understanding of the immediate consequences of a treatment decision (steps the patient must take to prepare in light of his/her treatment choice) and also projected longer term consequences (what the patient's life will be like a year after the decision). Because of these personal and projective aspects, appreciation (S3) has been difficult to model using standard neurocognitive measures (Dymek et al., 2001; Marson et al., 1996).

Both the baseline and baseline-6-month multivariate models of appreciation (S3) consisted solely of a measure of working memory/arithmetic (WAIS-III Arithmetic) (Tables 4 and 6). The 6-month model consisted solely of a basic executive function measure (Token Test). As reflected in achieved $R^{2} \mathrm{~s}$, all three models for S3 were notably weaker than corresponding models developed for the understanding (S5) and reasoning (S4) consent abilities. As noted above, the appreciation standard is less factually intensive than the understanding and reasoning standards. Participants with TBI are better able to draw upon, and apply, their existing fund of general and personal knowledge in responding to the appreciation (S3) test items. As noted previously, S3 continues to be more difficult to model using neuropsychological test measures.

Basic verbal memory and basic executive abilities are significant findings. It is not a simple matter of pass/fail but rather a matter of having a point or two be meaningful as a predictor. Thus, measures of basic abilities such as the Token Test might be useful as a supplemental measure in capacity evaluations in persons with TBI that may help to rule out individuals who are clearly incapable. However, for understanding the subtleties of TBI on neurocognitive functioning and capacity, medical decision-making capacity measures such as the CCTI might be more meaningful in guiding such determinations.

The dynamics of a traumatic brain injury are related to the demands of the task and how a person processes medical information. Shortly after a head injury, memory functions are particularly vulnerable (Jacobs \& Donders, 2008; McWilliams \& Schmitter-Edgecombe, 2008). Before a person with a head injury can begin to reason, demonstrate mental flexibility in thinking about medical options, and then integrate such information (i.e., exercise executive functioning and working memory) (Vallat-
Azouvi, Weber, Legrand, \& Azouvi, 2007), he or she has to have basic abilities to encode, retain, and recall new information about his or her medical situation. Having adequate verbal memory for relevant medical information is a critical aspect of medical decision-making early during the course of recovery. Executive abilities are more salient later during the course of recovery as the ability to integrate new information becomes more important than memory per se.

The study findings have clinical implications. First, both in conjunction with formal capacity measures and separately, specific neuropsychological test results can help support clinician judgments of competency in TBI. At the time of acute hospitalization, clinicians should carefully consider short-term verbal memory abilities as part of their assessment of current MDC. At 6-month follow-up, clinicians should increasingly consider executive function and working memory abilities in their decisional process. Perhaps the most valuable clinical finding, however, concerned the relationship of baseline executive abilities and working memory to prediction of MDC at 6-month followup. Predictive findings of this type help clinicians, during the time of acute injury, to begin to provide prognostic judgments to patients and family members concerning future recovery of MDC.

A clinical caveat is also indicated. The present findings illuminate contributions of several neurocognitive domains to decisional capacity in TBI. At the same time, judgments of capacity should not be founded solely, or even primarily, on neuropsychological test results. Neuropsychological test data by itself cannot be determinative of capacity questions, which involve issues of individual autonomy. A capacity judgment is ultimately a clinical judgment that draws upon a wide variety of evidence, including the clinician's interview of the patient and others, formal capacity measure results like the CCTI (if available), cognitive test results, and the clinician's experience. Thus, as reflected by the current study, however, the best neuropsychological evidence in capacity determinations involves those cognitive findings that have been empirically linked to capacity outcomes.

Several limitations of the study should be noted. The sample sizes of the healthy adult and TBI groups were relatively small. Larger samples are needed to replicate and confirm the current predictor findings. The current results should thus be interpreted cautiously. Another limitation concerns the use of hypothetical medical vignettes to test for consent capacity. Although the CCTI vignettes present real-life medical situations, making decisions based on hypothetical medical situations is arguably different from making real-life, personal medical decisions with real-life consequences. However, prior studies have consistently found clinical vignettes to be an effective and valid basis for assessing competency in individuals with dementia and other neurocognitive disorders (Fitten \& Waite, 1990; Marson, Schmitt, Ingram, \& Harrell, 1994). In addition, the standardized approach of the CCTI permits clinicians to obtain objective, norm referenced capacity data that can inform the clinician's judgment of competency.

In summary, treatment consent is an act of complex medical decision-making, and a higher order IADL, with important clinical, legal, and ethical consequences. This study represents an initial step towards developing a neurologic model of MDC capacity loss and initial recovery in persons with TBI. Short-term 
verbal memory appears to be strongly associated with impaired consent capacity in moderately and severely injured TBI patients at the time of inpatient hospitalization. As these patients experience cognitive and functional recovery in the period of posthospitalization, executive function and working memory abilities are associated with improved decisional capacity at 6-month follow-up.

\section{References}

Benton, A., \& Hamsher, K. (1989). Multilingual aphasia examination. Iowa City, IA: AJA Associates.

Benton, A. L., Hamsher, K. D. S., Varney, N. P., \& Spreen, O. (1983). Contributions to neuropsychological assessment. New York: Oxford University Press.

Berg, E. (1948). A simple objective treatment for measuring flexibility in thinking. Journal of General Psychology, 39, 15-22.

Bontke, C., Dolan, J., \& Ivanhoe, C. (1994). Should we withhold food from patients with a persistent vegetative state? Journal of Head Trauma Rehabilitation, 9, 62-69.

Centers for Disease Control and Prevention. (1997). Traumatic brain injury-Colorado, Missouri, Oklahoma, and Utah, 1990-1993. Morbidity and Mortality Weekly Report, 48, 8-11.

Childs, N., \& Cranford, R. (1997). Termination of nutrition and hydration in the minimally conscious state: Contrasting clinical views. Journal of Head Trauma Rehabilitation, 12, 70-78.

Cope, D. (1989). Legal and ethical issues in the psychopharmacologic treatment of traumatic brain injury. Journal of Head Trauma Rehabilitation, 4, 13-21.

Dymek, M., Atchison, P., Harrell, L., \& Marson, D. (2001). Competency to consent to treatment in cognitively impaired patients with Parkinson's disease. Neurology, 56, 17-24.

Dymek, M. P., Marson, D. C., \& Harrell, L. (1999). Factor structure of capacity to consent to medical treatment in patients with Alzheimer's disease: An exploratory study. Journal of Forensic Neuropsychology, 1, $27-48$.

Earnst, K., Marson, D., \& Harrell, L. (2000). Cognitive models of physicians' legal standard and personal judgments of competency in patients with Alzheimer's disease. Journal of the American Geriatrics Society, 48, 919-927.

Fitten, L. J., \& Waite, M. S. (1990). Impact of medical hospitalization on treatment decision-making capacity in the elderly. Archives of Internal Medicine, 150, 1717-1721.

Grant, D., \& Berg, E. (1948). A behavioral analysis of the degree of reinforcement and ease of shifting to new responses in a Weigl-type card sorting problem. Journal of Experimental Psychology, 38, 404-411.

Griffith, H. R., Dymek, M. P., Atchison, P., Harrell, L., \& Marson, D. C. (2005). Medical decision-making in neurodegenerative disease: Mild AD and PD with cognitive impairment. Neurology, 65, 483-485.

Grisso, T. (1986). Evaluating competencies: Forensic assessments and instruments. New York: Plenum Press.

Grisso, T., \& Appelbaum, P. (1995). A comparison of standards for assessing patients' capacities to make treatment decisions. American Journal of Psychiatry, 19, 149-166.

Hannay, D., \& Fischer, J. L. (2004). Neuropathology for neuropsychologists. In M. Lezak, D. Howieson \& D. Loring (Eds.), Neuropsychological assessment (4th ed., pp. 158-286). New York: Oxford University Press.

Heaton, R., Grant, I., \& Matthews, C. (1991). Comprehensive norms for an expanded Halstead-Reitan battery: Demographic corrections, research findings, and clinical applications. Odessa: Psychological Assessment Resources.

Hillier, S., Sharpe, M., \& Metzer, J. (1997). Outcomes 5 years post- traumatic brain injury (with further reference to neurophysical impairment and disability). Brain Injury, 11, 661-675.

Jacobs, M. L., \& Donders, J. (2008). Performance discrepancies on the California Verbal Learning Test-Second Edition (CVLT-II) after traumatic brain injury. Archives of Clinical Neuropsychology, 23, 113-118.

Jennett, B. (1997). A quarter century of the vegetative state: An international perspective. Journal of Head Trauma Rehabilitation, 12, 1-12.

Kapp, M. (1992). Geriatrics and the law: Patient rights and professional responsibilities. New York: Springer.

Kersel, D., Marsh, N., Havill, J., \& Sleigh, J. (2001). Neuropsychological functioning during the year following severe traumatic brain injury. Brain Injury, 283-296.

Leon-Carrion, J., Alarcon, J., Revuelta, M., Murillo-Cabezas, F., Dominguez-Roldan, J., Dominguez-Morales, M., et al. (1998). Executive functioning as outcome in patients after traumatic brain injury. International Journal of Neuroscience, 94, 75-83.

Levin, H., O'Donnell, V., \& Grossman, R. (1979). The Galveston Orientation and Amnesia Test: A practical scale to assess cognition after head injury. Journal of Nervous and Mental Disorders, 20, 694-705.

Lezak, M. D., Howieson, D. B., \& Loring, D. W. (2004). Neuropsychological assessment ( $4^{\text {th }}$ ed.). New York: Oxford University Press.

Marson, D. (2001). Loss of financial competency in dementia: Conceptual and empirical approaches. Aging, Neuropsychology, and Cognition, 8, $164-181$.

Marson, D., \& Briggs, S. (2001). Assessing competency in Alzheimer's disease: Treatment consent capacity and financial capacity. In S. Gauthier \& J. L. Cummings (Eds.), Alzheimer's Disease and Related Disorders Annual 2001 (pp. 1-25). London: Martin Dunitz Ltd.

Marson, D., Dreer, L., Krzywanski, S., Huthwaite, J., DeVivo, M., \& Novack, T. (2005). Impairment and partial recovery of medical decision making capacity in traumatic brain injury: A six month longitudinal study. Archives of Physical Medicine and Rehabilitation, 86, 889-895.

Marson, D., \& Ingram, K. (1996). Competency to consent to treatment: A growing field of research. Journal of Ethics, Law and Aging, 2, 59-63.

Marson, D., Strickland, A., Hethcox, A., DeVivo, M., Taylor, S., Krzywanski, S., et al. (2001). Assessing competency to consent to treatment in traumatic brain injury. Paper presented at the $21 \mathrm{st}$ Annual Conference of the National Academy of Neuropsychology, San Francisco, California.

Marson, D. C., Chatterjee, A., Ingram, K. K., \& Harrell, L. E. (1996), Toward a neurologic model of competency: Cognitive predictors of capacity to consent in Alzheimer's disease using three different legal standards. Neurology, 46, 666-672.

Marson, D. C., Cody, H. A., Ingram, K. K., \& Harrell, L. E. (1995). Neuropsychologic predictors of competency in Alzheimer's disease using a rational reasons legal standard. Archives of Neurology, 52, 955959

Marson, D. C., Ingram, K. K., Cody, H. A., \& Harrell, L. E. (1995). Assessing the competency of patients with Alzheimer's disease under different legal standards. Archives of Neurology, 52, 949-954.

Marson, D. C., Schmitt, F., Ingram, K. K., \& Harrell, L. E. (1994). Determining the competency of Alzheimer's patients to consent to treatment and research. Alzheimer's Disease and Associated Disorders, 8(Suppl. 4), 5-18.

Mattson, A., \& Levin, H. (1990). Frontal lobe dysfunction following closed head injury: A review of the literature. Journal of Nervous and Mental Disease, 178, 282-290.

McAllister, T., Flashman, L. S., MB, \& Saykin, A. (2004). Working memory deficits after traumatic brain injury: Catecholaminergic mechanisms and prospects for treatment: A review. Brain Injury, 18, 331350 .

McDonald, B., Flashman, L., \& Saykin, A. (2002). Executive dysfunction following traumatic brain injury: Neural substrates and treatment strategies. NeuroRehabilitation, 17, 333-344. 
McDowell, S., Whyte, J., \& D'Esposito, M. (1997). Working memory impairments in traumatic brain injury: Evidence from a dual task paradigm. Neuropsychologia, 35, 1341-1353.

McWilliams, J., \& Schmitter-Edgecombe, M. (2008). Semantic memory organization during the early stage of recovery from traumatic brain injury. Brain Injury, 22, 243-253.

Moye, J., Karel, M., Gurrera, R., \& Azar, A. (2006). Neuropsychological predictors of decision-making capacity over 9 months in mild-to-moderate dementia. Journal of General Internal Medicine, 21, 78-83.

Mukherjee, D., \& McDonough, C. (2006). Clinician perspectives on decision-making capacity after acquired brain injury. Topics in Stroke Rehabilitation, 13, 75-83.

Okonkwo, O., Griffith, H., Belue, K., Lanza, S., Zamrini, E., Harrell, L., et al. (2008). Cognitive models of medical decision-making capacity in patients with mild cognitive impairment. Journal of International Neuropsychological Society, 14, 297-308.

Phipps, E. (1998). Communication and ethics: Cardiopulmonary resuscitation in head trauma rehabilitation. Journal of Head Trauma Rehabilitation, 13, 95-98.

Ponsford, J., Draper, K., \& Schonberger, M. (2008). Functional outcome 10 years after traumatic brain injury: Its relationship with demographic, injury severity, and cognitive and emotional status. Journal of the Neuropsychological Society, 14, 233-242.

Proctor, A. W., B, Sanchez, C., \& Wesley, E. (2000). Executive function and verbal working memory in adolescents with closed head injury (CHI). Brain Injury, 14, 633-647.

Rao, S. M. (1990). A manual for the brief repeatable battery of neuropsychological tests in multiple sclerosis. New York: National Multiple Sclerosis Society.

Reid-Proctor, G., Galin, K., \& Cummings, M. (2001). Evaluation of legal competency in patients with frontal lobe injury. Brain Injury, 15, 377386

Reitan, R., \& Wolfson, D. (1993). The Halstead-Reitan neuropsychological test battery: Theory and clinical interpretation. Tucson, AZ: Neuropsychology Press.

Rey, A. (1964). L'examen clinique en psychologie. Paris: Presses Universitaires de France.

Rosenthal, M., \& Ricker, J. (2000). Traumatic brain injury. In R. Frank \& T. Elliott (Eds.), Handbook of rehabilitation psychology (pp. 49-74). Washington, DC: American Psychological Association.
Roth, L., Meisel, A., \& Lidz, C. (1977). Tests of competency to consent to treatment. American Journal of Psychiatry, 134, 279-284.

Royall, D., Mahurin, R., \& Gray, K. (1992). Bedside assessment of executive cognitive impairment: The executive interview. Journal of the American Geriatrics Society, 40, 1221-1226.

Simon, R. (1994). Ethical and legal issues. In J. M. Silver, S. D. Yudofsky, \& R. E. Hales (Eds.), Neuropsychiatry of traumatic brain injury (pp. 569-627). Washington, DC: American Psychiatric Association.

Smith, A. (1982). Symbol Digit Modalities Test (SDMT). Manual (Revised). Los Angeles: Western Psychological Services.

Taylor, E. (1959). Psychological appraisal of children with cerebral deficits. Cambridge, MA: Harvard University Press.

Teasdale, G., \& Jennett, B. (1974). Assessment of coma and impaired consciousness. Lancet, 2, 81-84.

Tepper, A., \& Elwork, A. (1984). Competency to consent to treatment as a psychological construct. Law and Human Behavior, 8, 205-223.

Vakil, E. (2005). The effect of moderate to severe traumatic brain injury (TBI) on different aspects of memory: A selective review. Journal of Clinical and Experimental Neuropsychology, 27, 977-1021.

Vallat-Azouvi, C., Weber, T., Legrand, L., \& Azouvi, P. (2007). Working memory after severe traumatic brain injury. Journal of the International Neuropsychological Society, 13, 770-780.

Wechlser, D. (1997). Manual for the Wechsler Adult Intelligence ScaleThird Edition. San Antonio, TX: The Psychological Corporation.

Wechsler, D. (1981). Wechsler Adult Intelligence Scale-Revised. New York: The Psychological Corporation.

Wechsler, D. (1987). Manual for the Wechsler Memory Scale-Revised. San Antonio, TX: The Psychological Corporation.

Wong, J., Clare, C., Gunn, M., \& Holland, A. (1999). Capacity to make health care decisions: Its importance in clinical practice. Psychological Medicine, 29, 437-446.

Wong, T. (1998). Ethical issues in the evaluation of treatment of traumatic brain injury. In Avoiding ethical misconduct in psychology specialty areas (pp. 187-200). Springfield, IL: Charles C. Thomas.

Zink, B. (2001). Traumatic brain injury outcome: Concepts for emergency care. Annals of Internal Medicine, 37, 318-332.

Received December 10, 2007

Revision received March 25, 2008

Accepted June 22, 2008 